\title{
Umbilical Cord Blood Transplantation
}

National Cancer Institute

\section{Source}

National Cancer Institute. Umbilical Cord Blood Transplantation. NCI Thesaurus. Code C15640.

A therapeutic procedure that involves the transplantation of hematopoietic stem cells collected from the umbilical cord or placenta. 